\title{
Lesioni vascolari e nervose in chirurgia ortopedico traumatologica
}

\author{
Paolo Ghiggio ${ }^{1, a}(\bowtie)$, Elvio Novarese ${ }^{2, b}$ \\ ${ }^{1}$ Ortopedia e Traumatologia-Chirurgia della mano, ASLTO4 Ivrea Cuorgnè Ciriè, Ivrea (To), Italia \\ ${ }^{2}$ Ortopedia e Traumatologia, ASLTO5 Chieri, Chieri (To), Italia \\ apaologhiggio@virgilio.it; belvionovarese@yaohho.it \\ Pubblicato online: 11 marzo 2014 \\ (C) Springer-Verlag Italia 2014
}

Quello delle lesioni iatrogene in chirurgia ortopedico-traumatologica è stato un argomento già oggetto di pubblicazioni in passato. I lavori in letteratura, però, si sono occupati di tale argomento settorialmente e, a volte, in modo dispersivo. Lo scopo di questa monografia è quello di raggruppare in un unico volume le possibili lesioni iatrogene nell'approccio chirurgico dei vari distretti e nelle varie patologie di interesse sia ortopedico sia traumatologico. Troverete infatti i capitoli dedicati al rachide, curatori i colleghi Eugenio Boux e Vittorio Salvi, quello dell'arto superiore, curatore Paolo Ghiggio, il settore del bacino, seguito da Roberto Ramella, l'arto inferiore, curato da Angelo Dettoni. Un capitolo a parte è quello delle problematiche medico legali, che chiarirà le idee sulla differenza fra complicanza ed errore medico.

Con queste premesse speriamo di avere fatto cosa utile soprattutto per i giovani colleghi che si affacciano a questa affascinante chirurgia. Leggendo queste pagine e apprendendo i segreti da colleghi esperti potranno meglio navigare in queste acque insidiose, con l'augurio di evitare o perlomeno limitare i contenziosi medico legali, ormai dilaganti nella nostra professione.

Da queste righe giunga il ringraziamento ai colleghi piemontesi sia ospedalieri sia universitari, che hanno dato la loro disponibilità, collaborando con la SOTOP, alla stesura di questa monografia.

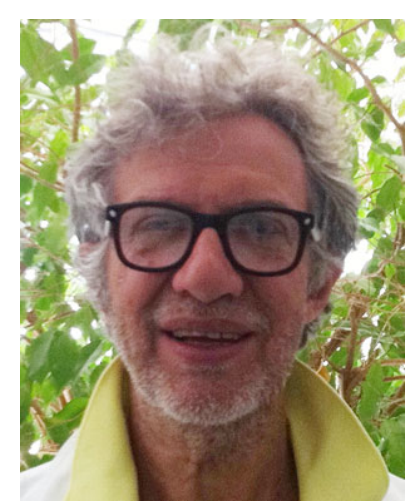

Paolo Ghiggio

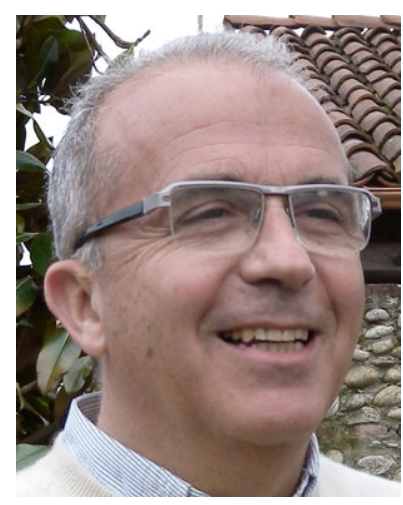

Elvio Novarese

Infine un grazie ai direttori scientifici de Lo Scalpello, che hanno voluto affidarci questa fatica, proseguendo con OTODI nella tradizione regionale di distribuzione dei fascicoli. 